\title{
Automatisierung von Prozessmodulen
}

\section{Von Package-Unit-Integration zu modularen Anlagen}

In Verfahrenstechnik und Anlagenbau werden derzeit Konzepte zur Standardisierung und Modularisierung diskutiert. Dies hat weitreichende Auswirkungen auf das Engineering und den Betrieb der Anlagen. In diesem Beitrag werden die Analyse des NamurArbeitskreises 1.12 und die daraus resultierenden Herausforderungen an die Automatisierungstechnik vorgestellt. Zusätzlich diskutieren die Autoren erste Lösungsansätze, die sich aus heutigen Erfahrungen mit der Integration von Package-Units ergeben.

SCHLAGWÖRTER Modularisierung / Package-Unit Integration / Automatisierungsengineering

\section{Automation of Modules -}

\section{From Package-Unit-Integration towards Modular Plants}

Standardisation and modularisation are intensively discussed in the Process Industries. Introducing these concepts will dramatically change plant engineering processes as well as plant operation strategies. This paper presents the results of an in-depth analysis of the expected impact on process automation which was conducted by the Namur working group 1.12. Experiences from package-unit integration provide a solid ground to sketch first solutions for the challenges given by modular plant concepts.

KEYWORDS Modular Plants / Package-Unit Integration / Engineering of Industrial Automation 


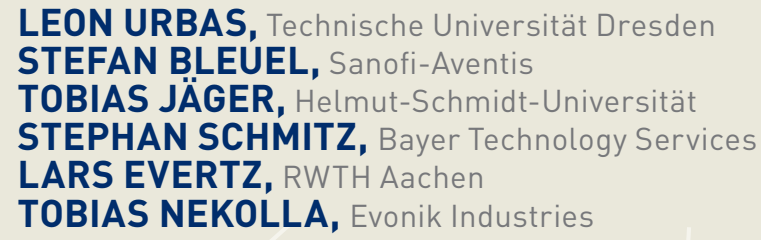

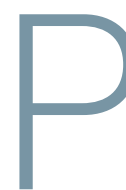

roduktionsanlagen werden zumeist um einen oder mehrere Referenzprozesse herum ausgelegt. Die für die Anlagenautomatisierung benötigten Feldgeräte, Hardwarekomponenten, und Softwarebausteine werden dabei mit dem Ziel ausgewählt und gestaltet, eine technisch und wirtschaftlich optimierte Prozessführung zu erreichen. Hierzu wird für jede neue Anlage ein mehr oder weniger vollständiger Engineeringprozess durchgeführt, um bei vorgegebenen Produktspezifikationen und Sicherheitsstandards die anlagenspezifischen Investitions- und Betriebskosten zu minimieren. Dieses zeitaufwendige Vorgehen erklärt sich aus der hohen Individualität der Anlagen, der Anpassung an örtliche Gegebenheiten und den langen projektierten Laufzeiten. Ausnahmen von dieser Vorgehensweise sind bei Package-Units zur Versorgung mit Einsatzund Hilfsstoffen oder zur Konfektionierung von Endprodukten zu beobachten.

Dieses Vorgehen ist für bestimmte Produkte aufgrund der benötigten Zeit zwischen Produktidee und produzierender Anlage nicht mehr wettbewerbsfähig. Dies gilt vor allem für Projekte, bei denen eine Spanne von mehr als drei Jahren zwischen Investitionsentscheidung und Markteintritt ein erhebliches Risiko darstellt. Als wichtigste Hebel für die Verkürzung der Planungsprozesse hat die Prozessindustrie weitergehende Standardisierung, integrierte Informationsflüsse und Arbeitsprozesse sowie die Verwendung von Modulen identifiziert [1]. Im Idealfall ersetzt ein Auswahlprozess den Auslegungsprozess. Dabei wird einzig die Frage beantwortet, ob die geforderte Prozessfunktion mit einer bestehenden Modulbaugruppe wirtschaftlich umsetzbar ist (Bild 1).

\section{MODULARE ANLAGEN}

Der Nutzen eines beschleunigten Verfahrens für Konzeption, Engineering, Aufbau und Inbetriebnahme von Anlagen lässt sich anhand des zeitlichen Verlaufs des kumulierten Finanzflusses (Cumulated Cash Flow, CCF) darstellen. Bild 2 zeigt die qualitativen Verläufe des CCF beim klassischen Engineering (blaue Linie) und bei der Verwendung eines modularen Anlagenkonzepts (rote Linie). Der Tiefpunkt liegt jeweils nach Inbetriebnahme der Anlage. Es wird deutlich, dass allein durch eine zeitlich stark nach vorne gerückte Inbetriebnahme der modularen Anlage trotz gegebenenfalls höherer Investitionskosten und geringerer Effizienz in einem bestimmten Zeitintervall ein höherer positiver CCF erzielt werden kann. Die Wirtschaftlichkeit modularer Anlagenkonzepte ist durch vier grundsätzliche Kenngrößen gekennzeichnet:

1| Verkürzung der Dauer von Start bis Inbetriebnahmezeitpunkt: je früher, desto besser.

2 | Veränderung der Investitionskosten: Das entspricht näherungsweise dem maximalen negativen CCF. Bei einer modularen Realisierung sind Mehrkosten durch die notwendige Überdimensionierung und kostenreduziernde Skaleneffekte durch höhere Stückzahlen denkbar.

3| Qualitativer Verlauf des Cashflow nach Inbetriebnahme: je steiler die Steigung der Kurve nach Inbetriebnahme, desto besser.

4 | Erwartete Lebensdauer des Produkts beziehungsweise der Anlage

Unterschiedliche Modularisierungskonzepte beeinflussen verschiedene Kenngrößen. Eine Verringerung der projektspezifischen Planungsaufwände kann den Inbetriebnahmezeitpunkt nach vorne schieben. Dies wird beispielsweise durch Modularisierung und Wiederverwendung bewährter Lösungen erreicht. Die Optimierung des positiven Cashflows nach Inbetriebnahme ergibt sich durch eine Anpassung der Apparate und der Prozessführungsstrategie an die projektspezifischen Gegebenheiten.

Daraus entsteht ein Zielkonflikt: Eine optimal angepasste Anlage mit maximaler Ausbeute und minimalen 
Modulares

Engineering

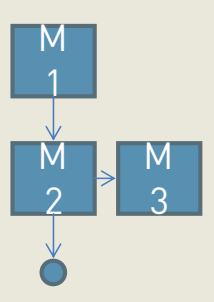

Prozesssynthese, Standort,

Wirtschaftlichkeit Prozessführung

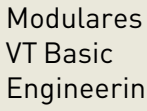

\section{Modulares \\ P\&IDBasic \\ Engineering}

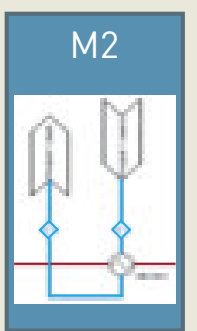

HAZOP,SIL,

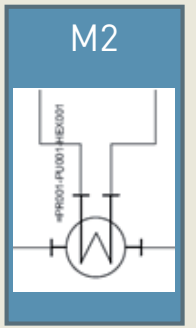

Auswahl Ausführungs varianten
Modulares

P\&IDDetail

Engineering

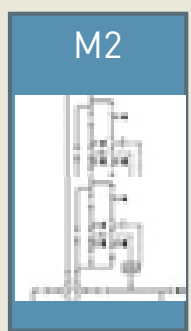

Parametrierung derModule und desEnsembles

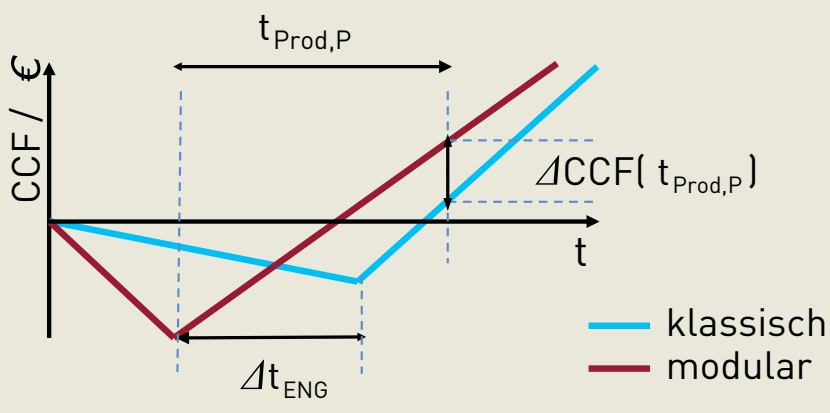

CCF: $\quad$ Cumulated Cash Flow

$\triangle \mathrm{t}_{\mathrm{ENG}}$ : Verkürzung der Projektlaufzeit

$\triangle \mathrm{CCF}\left(\mathrm{t}_{\mathrm{Prod}, \mathrm{P}}\right)$ : Veränderung des CCF nach planmäßiger Laufzeit der Anlage

BILD 1: Modulares Engineering in Anlehnung an [5]

BILD 2: Kumulierter Finanzfluss bei klassischem Engineering (blaue Linie) und beschleunigtem Engineering auf Basis modularer Anlagen (rote Linie)
Betriebskosten erfordert einen hohen Engineeringaufwand, der sich in einem späten Inbetriebnahmezeitpunkt bemerkbar macht. Eine Planung mit vordefinierten Modulen eines Baukastensystems führt zu Anlagen, die sehr schnell in Betrieb gehen können, zumeist jedoch höhere spezifische Betriebskosten aufweisen.

Um diesen Zielkonflikt zu lösen, ist es erforderlich, die Planungsprozesse zu verändern. Diese orientieren sich heute an dem gesteckten Investitionsbudget und stellen eine optimale Anlage sicher. Betriebskosten werden für einzelne Positionen mit einem hohen Betriebskostenanteil wie beispielsweise Verdichter oder Katalysatoren berücksichtigt.

\section{MODULVARIANTEN}

Aus den in der Verfahrenstechnik diskutierten und vorangetriebenen Ansätzen zur Modularisierung, angefangen von Conceptional Design [1] bis zu Planung und Konstruktion [2], hat der Namur-Arbeitskreis 1.12 „Anforderungen an die Automatisierungstechnik durch die Modularisierung verfahrenstechnischer Anlagen“ für seine Analysen den Ansatz der $\mathrm{F}^{3}$-Factory [3] ausgewählt. Dieser Ansatz geht davon aus, dass Module für eine vorhandene Infrastruktur ausgelegt sind und zum Aufbau einer Produktionsanlage lediglich „gesteckt“ werden (siehe Bild 3). Dabei sind aus Sicht der Automatisierungstechnik drei Modulvarianten sinnvoll:

1 | Variante Stand-Alone: Alle Automatisierungsfunktionen werden mit dem Modul geliefert. Jede Box wird autonom betrieben und ist mit einer MenschMaschine-Schnittstelle (HMI) ausgestattet. Eine automatisierungstechnische Verbindung der Module ist nicht vorgesehen. Gegebenenfalls erfolgt eine Datenübergabe an ein Betriebsdatenerfassungssystem (BDE).

2 | Variante Integrierte Box: Die fest definierten und im Wesentlichen unveränderlichen Module sind über eine Einrichtung stofflich, energetisch und automatisierungstechnisch verbunden. Das erlaubt modulübergreifende Verriegelungen, Regelkreise und Rezeptfahrweisen. Alle Module werden über ein zentrales HMI gefahren.

3 | Variante Flexible Box: Bei dieser Spielart wird Flexibilität innerhalb des Moduls realisiert. Equipment und Apparate des Moduls können an die verfahrens- und automatisierungstechnischen Anforderungen angepasst werden. Dieser Ansatz ist $\mathrm{F}^{3}$-Factory [3] vorgesehen. Erfolgt diese Anpassung in einem modularisierten Baukastensystem, decken sich die automatisierungstechnischen Betrachtungen für diese Variante mit denen der integrierten Box.

Alle drei Varianten beschleunigen den Anlagenplanungsprozess durch kürzere Engineeringzeiten, Vorfertigung, Transportierbarkeit und Numbering Up statt Scale Up. In diesem Beitrag wird lediglich die Variante integrierte Box betrachtet, da sich an dieser die automatisierungstechnische Perspektive auf eine verfahrenstechnisch motivierte Modularisierung vollständig diskutieren lässt. 


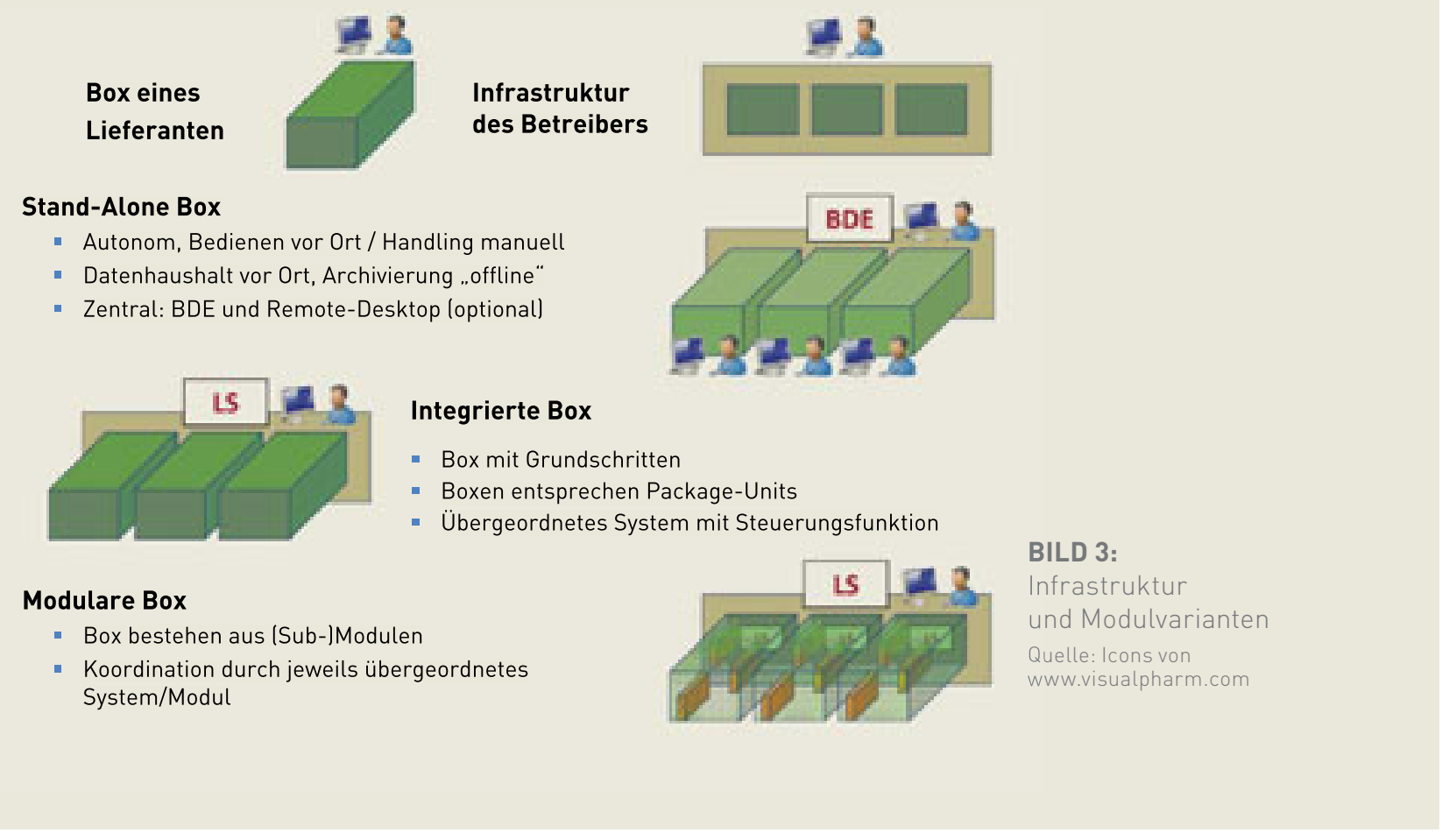

\subsection{Integrierte Box}

Aus automatisierungstechnischer Sicht ist das wesentliche Merkmal einer integrierten Box die Möglichkeit, modulübergreifende Steuerungen in einem übergeordneten System zu realisieren. Wenn nur Module verwendet werden, deren Schnittstellen und Fahrweisen (in IEC 61512/ISA S88 [4] als State bezeichnet) im Automatisierungssystem bekannt sind, dann lassen sich Schrittketten (IEC 61512: Phases, Operations, Procedures) ohne weiteres modulübergreifend verwirklichen. Das gilt auch für vollständige Rezepte. Voraussetzung ist, dass ein im Automatisierungssystem hinterlegter Softwarebaustein die Logik des Moduls beschreibt. Durch die Gesamtheit aller im System hinterlegten Softwarebausteine ergibt sich ein Modulkatalog, aus dem vor Inbetriebnahme ein Abbild der realen Anlage durch Verknüpfen der einzelnen Module - vergleichbar mit der grafischen Programmierung mit Funktionsbausteinen - geschaffen werden muss.

Beim Anschließen der verfahrenstechnischen Module an eine gegebene Infrastruktur muss durch Abfrage der Moduleigenschaften und Vergleich mit den im Modulkatalog des Automatisierungssystems beschriebenen Merkmalen ein Plausibilitätscheck durchgeführt werden, der ergeben muss, dass die Zuordnung des Moduls auf eine bestimmte Dockingstelle kongruent zum Anlagenbild ist. Auf Steuerungsseite erstreckt sich der Plausibilitätscheck auf die Existenz benötigter Fahrweisen und ebenso auf die Instrumentierung, da bestimmte Messwerte für übergeordnete Regelungen und Schrittketten vorhanden sein müssen. Für die Prozessoptimie- rung und den Betrieb qualifizierter Prozesse wird zudem eine eindeutige Identifizierung des Moduls notwendig sein. Dies kann durch die Speicherung einer ID-Nummer geschehen, die ebenfalls über diese Schnittstelle auslesbar sein muss. Um die geforderte Zeiteinsparung zu erreichen, muss es möglich sein, die verschiedenen Bausteine für den automatisierten Ensemblebetrieb zu parametrieren, ohne auf Engineering-Ebene einzugreifen. Insbesondere müssen die Alarmgrenzen und Schaltwerte der Module einstellbar sein. Dies ist im aktuellen Stand der Technik ohne Weiteres umsetzbar.

Es ist jedoch davon auszugehen, dass die Sicherheitsbetrachtung einer modular aufgebauten Anlage eine Einzelfallbetrachtung bleiben wird, da hierfür die (vorgegebenen) Schutzeinrichtungen der Module mit den (individuell) gehandhabten Stoffen kombiniert werden müssen. Eine besondere Herausforderung stellen dabei modulübergreifende Sicherheitskreise dar. Es ist zwar technisch möglich, den einzelnen Modulsteuerungen Hardware-Signale benachbarter Module zur Verfügung zu stellen. Für die weiteren Betrachtungen wird jedoch angenommen, dass die einzelnen Module funktional eigensicher sind. Modulinterne Maßnahmen verhindern eine Fehlerfortpflanzung.

\section{MODULARE AUTOMATISIERUNGSSTRUKTUREN}

Aus der Erfahrung mit der Implementierung verteilter Automatisierungssysteme und den Schwierigkeiten und Aufwänden bei der Integration von Package-Units hat der Arbeitskreis die in Bild 4 dargestellte modulare Automa- 
tisierungsstruktur entworfen. Diese Architektur geht davon aus, dass Module eine eigene Automatisierung besitzen und über wesentliche für den Anlagenbetrieb notwendige Informationen Auskunft geben können. Dazu gehören dynamische Daten über den Prozesszustand im Modul, statische Information über Bedienzugänge, lokale Rezepte, Verriegelungen und leittechnische Schutzeinrichtungen.

\subsection{Automatisierung des Moduls}

Das Automatisierungssystem des Moduls umfasst Sensoren, Aktoren, Signalübertragung, Feldbussysteme, Ex-Barrieren, Signalumwandlung und Verkabelung. Optional ist ein Modul mit einer eigenständigen Steuerung zur Implementierung von modulspezifischen Steuerungs- und Regelungsalgorithmen ausgestattet, die auch die funktionale Eigensicherheit des Moduls gewährleisten. Für den Batchbetrieb sind auf der Modulsteuerung zudem Grundfunktionen für die Rezeptsteuerung implementiert.

Die Auswahl geeigneter Messprinzipien, der Geräte und deren Auslegung liegen im Verantwortungsbereich des Modulherstellers. Das gleiche gilt für die Konzeption, Auslegung und Implementierung der Signalübertragung und der Steuerung. Für Module, die in explosionsgefährdeten Bereichen [6] eingesetzt werden oder in denen Ex-Bereiche definiert sind, ist ein Schutzkonzept $\mathrm{zu}$ realisieren und gegenüber dem Modulbetreiber, beispielsweise durch die ATEX-Zertifikate der verbauten Produkte, zu belegen.

Für die Integration in das übergeordnete Automatisierungssystem müssen Konventionen bei der Vergabe von TAG-Nummern beachtet werden. Jedem Sensor, Aktor und anderen Feldgeräten (wie Schalter/Taster, Leuchte, Anzeiger) des Moduls ist eine eineindeutige TAG-Nummer zuzuordnen, zum Beispiel nach DIN EN 61346 [7]. Im Kontext der Modularisierung ist zu beachten, dass die Integration in ein übergeordnetes Automatisierungssystem weitere Anforderungen an die Vergabe von TAGs stellt, damit beispielsweise die Betreiber von Produktionsanlagen ihre rechtlichen Nachweispflichten erfüllen können.

\section{Steuerung}

Schutzfunktionen, die mit Mitteln der Prozessleittechnik realisiert werden, gehören zum Automatisierungssystem des Moduls. Modulübergreifende Schutzfunktionen sollen auf dieser Ebene vermieden werden.

Für die Anwendersoftware sind die gültigen Normen und Richtlinien zu beachten. Gegebenenfalls ist ein lokales HMI-System für das Bedienen und Beobachten und ein Archiv vorzusehen. Das beinhaltet folgende Punkte:

- Fließbilder mit dynamischen Anzeigen von

Prozess- und Gerätezuständen

- Darstellung von Verriegelungen

- Trendkurven und Trendkurvengruppen

- Alarm- / Meldesystem

Für die Darstellungen und Bedienungen gelten die gültigen Normen und Richtlinien. Die Datenhaltung (Persistenz) der Anwendersoftware sowie der Prozessdaten und Parameterwerte ist innerhalb des Systems zu realisieren. Ein lokales Engineeringsystem beziehungsweise ein geeigneter Zugang für Parametrierung und Diagnose des Moduls gehört zum Lieferumfang. Von Vorteil ist eine Integration dieser Aktivitäten in das Engineeringsystem des übergeordneten Automatisierungssystems.

\section{2 Übergeordnete Automatisierung}

Wie im vorherigen Abschnitt ausgeführt, sind in dem vorgestellten Konzept die leittechnischen Funktionen in den Automatisierungssystemen der Module realisiert und
BILD 4:

Architektur eines

Automatisierungssystems für modulare Anlagen

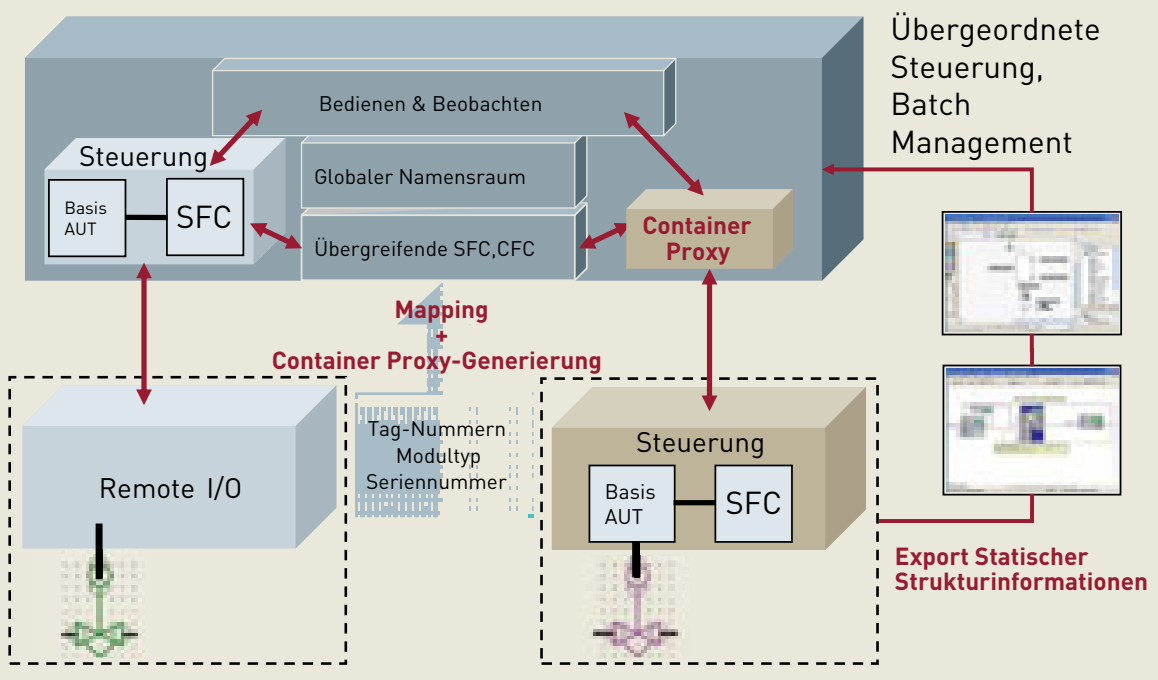


werden dort bearbeitet. Mit dem übergeordneten Automatisierungssystem erfolgt das Bedienen und Beobachten für alle angeschlossenen Module. Die wesentlichen Aufgaben dieses Automatisierungssystems:

- Vergabe von eineindeutigen TAG-Nummern für die Feldgeräte in den angeschlossenen Boxen, sofern sie im übergeordneten Automatisierungssystem relevant sind

- Parametrieren der Softwarebausteine, die die Modulautomatisierung repräsentieren

- HMI für die Visualisierung der Prozesse in den Modulen mit einheitlicher Bedienung \& Beobachtung

- Datendrehscheibe für Systeme wie PIMS, LIMS oder MES

- Diagnose der angeschlossenen Boxen

\section{Vergabe von TAG-Nummern:}

Aus den TAG-Nummern des Moduls sollen nach einem parametrierbaren Algorithmus die TAG-Nummern für das übergeordnete Automatisierungssystem automatisiert abgeleitet werden, sodass die Feldgeräte im übergeordneten Automatisierungssystem eineindeutig benannt sind.

\section{Einheitliches Bedienen und Beobachten}

Beim übergeordneten Automatisierungssystem kommt es auf einheitliches Bedienen und Beobachten des durch die Module realisierten Prozesses an. Das bezieht sich auf Darstellung und Bedienkonzept in den Bedien- und Anzeigebildern (Faceplates) und in den Fließbildgrafiken. Konkret heißt das, dass Faceplates für Motoren, Ventile, Regler und die grafischen Elemente in den Fließbildgrafiken für alle Module gleich zu gestalten sind. Ein Ansatz, der eine automatische firmenspezifische Anpassung von Darstellung und Bedienkonzept ermöglicht, ist die automatische Generierung von Fließbildgrafiken aus einem neutralen Datenformat [13]. Sofern in den Modulen Schrittketten, eventuell auch im Rahmen von Rezepturen realisiert sind, ist für das gesamte Ensemble ebenfalls auf eine einheitliche Darstellung und Bedienung zu achten. Gleiches gilt für Verriegelungen sowie Trendkurven und Trendkurvengruppen. Folgende Funktionen sind übergreifend umzusetzen:

- Einheitliche Systemzeit, Synchronisation mit den Automatisierungssystemen der Module

- Alarm- und Meldesystem

- Archivierung von Prozessdaten

- Zugriffssystem

Für die Darstellungen und Bedienungen sind die gültigen Normen und Richtlinien zu beachten.

\section{Datendrehscheibe}

Mit der beschriebenen Systematik sind im übergeordneten Automatisierungssystem alle relevanten Daten der Module vorhanden. Für die Betriebs- und Unternehmensführung notwendige Systeme wie PIMS, LIMS oder MES können die Daten der Module somit aus dem Datenhaushalt des übergeordneten Automatisierungssystems ab- greifen. Übernimmt dieses Automatisierungssystem dabei die Funktion einer Firewall, so leistet das vorgestellte Architekturkonzept einen Beitrag zur IT-Sicherheit in der Automatisierung mithilfe von definierten lokalen und anlagenweiten Sicherheitszonen und verwalteten und überwachbaren Übergängen nach IEC 62443 [8].

Die Aufgaben der Prozessführungsebene erfordern darüber hinaus, dass das übergeordnete System Daten für die Diagnose der Module erhält. Zur Unterstützung von Fehlerlokalisierung, -diagnose und -behebung müssen dort Systemzustände des Moduls angezeigt werden können, die detailliert Auskunft über die Funktionsweise und Funktionsfähigkeit des Moduls geben.

Die hier aufgeführten dynamischen Daten müssen in Echtzeit zwischen den Modulen und dem übergeordneten System kommuniziert werden. Weiterer Kommunikationsbedarf besteht für die statischen Strukturdaten der Modulautomatisierung, die für die Planungs- und Inbetriebsetzungsschritte nötig sind.

\section{MODULARES ENGINEERING}

\subsection{Auswahl}

Geht man davon aus, dass es sich bei der modularen Einheit um eine Teilanlage handelt, dann sind für den Auswahlprozess zunächst folgende, grundsätzliche Fragestellungen relevant:

匹 Sind die zur Prozesssführung notwendigen Signale und (Regelkreis-) Strukturen vorhanden?

- Sind die zur Teilanlagenüberwachung benötigten Signale (Meldungen) über die Unitgrenze hinweg verfügbar?

- Sind Tuning-Parameter sowie Verriegelungen und Alarmgrenzen in geeigneter Weise konfigurierbar?

- Sind die implementierten Schrittketten geeignet, das Herstell-Rezept umzusetzen?

Um diese Fragen beantworten zu können, wird der Planer auf die Dokumentation des Herstellers zurückgreifen und diese auch daraufhin prüfen müssen, ob sie sich eignet, die Anlagen- und Produktsicherheit nachzuweisen. Auch dieser Gedanke ist ein Plädoyer für die Auswahl von Standardlieferanten, da sonst durch ein langwieriges Studium und die Bewertung der Dokumentationsvielfalt der Zeitvorteil des Planungsprozesses modularer Anlagen schrumpfen wird.

Bei einer umfassenden Dokumentation hoher Güte wird der Planer mitunter nicht umhinkommen, den Hersteller in die Maßnahmenbewertung der Risikoanalysen zur Produkt- und Anlagensicherheit einzubeziehen. Dies muss aus technischer Sicht kein Nachteil sein, jedoch werden dadurch große Teile eines Prozesses einem Dritten offenbart.

\subsection{Konfektion und Integration}

Unter Integrationsengineering werden im Rahmen der modularisierten Automatisierung die Aufgaben bei der 
Integration verschiedener Module zu einem Gesamtsystem verstanden. Das Integrationsengineering ist stark von der Nutzung vorhandener (oder noch zu entwickelnder) Schnittstellenstandards der Module und des übergeordneten AS sowie der eingesetzten Engineering-Werkzeuge abhängig. Dabei übernimmt das Integrationsengineering die Schlüsselfunktion zur Festlegung der zu nutzenden Schnittstellen und damit auch der Aufgabenverteilung innerhalb der Automatisierungsstruktur.

Neben den automatisierungstechnischen Schnittstellen zwischen den Modulen und einem übergeordneten AS müssen die planungstechnischen Informationen und der spätere Betrieb der Anlage berücksichtigt werden. Idealerweise wird über das Integrationsengineering mithilfe einer Kommunikationsplattform (übergeordnetes AS) der Datenaustausch zwischen den Modulen und zu weiteren Automatisierungssystemen (MES, LIMS) mit standardisierten Schnittstellen sichergestellt.

Defizite in der Bereitstellung standardisierter Schnittstellen und unterschiedliche Standards in der Automatisierung der Module führen zu entsprechendem Aufwand bei der Integration der Module zu einem Gesamtsystem. Je nach Einsatzmöglichkeit von Standards bei der Modulautomatisierung sowie dem Engineering lassen sich Datenimporte aus dem Fundus der Modulhersteller zur Automatisierung über das übergeordnete AS nutzen. Heute noch auftretende Defizite in der Verfügbarkeit von Engineeringdaten der Hersteller müssen durch entsprechende Konfigurationsmöglichkeiten des übergeordneten AS oder gegebenenfalls der Kommunikationsplattform ausgeglichen werden:

- Unterschiedliche Datenformate

- Physikalische Einheiten, Wertigkeit,

Arbeitsstrom / Ruhestrom

- Übertragungsraten

- Asynchrone Daten, Zeitstempel

- TAG-Nummern, Bezeichnungen

- Grafikbilder

- Objekteigenschaften

(auch für Darstellungen von Geräten)

- Bedeutung und Interpretation von Fehlerzuständen einzelner Signale oder Aggregate

Hinzu kommen noch spezielle Aufgaben des Integrationsengineerings, um eine Bedienung der Gesamtanlage zu ermöglichen. Zum Beispiel:

- Einheitliches Zugriffssystem (single sign on) für alle Aggregate

- Einheitliches Look and Feel für die verschiedenen Module auf dem übergeordneten AS

- Einheitliche Bedienoberfläche / Funktionen, zumindest bei Bedienung über das übergeordnete AS

- Lastverteilung zwischen den Modulen

- Organisation der Module im Rahmen einer Rezeptfahrweise

- Überwachung von Ver- und Entsorgungseinrichtungen zur Vermeidung der Überlastung durch Gleichzeitigkeit

- Modulübergreifende Advanced Process Control Strategien
- Einbindung von Systemen übergeordneter Führungsebenen wie MES, ERP, LIMS

- Verwalten der angedockten Module und deren Betriebszustände

- Umgang mit historischen Konfigurationsständen

- Berücksichtigung sicherheitsrelevanter Funktionen

- Modulübergreifende Prozeduren müssen beim Engineering entworfen werden

Aufgrund der gewollten Flexibilität in der Ausgestaltung der einzelnen Module besteht ein Risiko für unvorhersehbare Wechselwirkungen an den technischen Schnittstellen des Moduls zur übergeordneten Anlage. Diese wirken sich erstmalig im Rahmen der Inbetriebnahme aus. Fehlende oder fehlerhaft implementierte Funktionen können zum Ausfall des übergeordneten Systems führen. Aufgrund der stofflich-energetischen Kopplung können zudem unerwünschte Wechselwirkungen zwischen den Modulen auftreten.

Die Wahl und Berücksichtigung einer geeigneten Standardisierung, hinsichtlich Hard-/Software, Informationsaustausch und -offenheit, ist ein Mittel, diesen Risiken entgegenzuwirken. Rückwirkungsfreiheit und Funktionsrisiken müssen im Rahmen der Tests geprüft werden.

\subsection{Dokumentation}

Ein wesentlicher Aspekt eines Projekts liegt in der Dokumentation. Zur Stärkung der Kommunikation und zur Vermeidung von Fehlinterpretationen müssen die verwendeten Begrifflichkeiten beispielsweise durch ein Glossar definiert werden. Ebenfalls sind die zur Integration notwendigen Informationen festzuhalten. Das gilt insbesondere für die Schnittstellenbeschreibungen. Diese Aspekte sind eindeutig und nach den Notwendigkeiten der beteiligten Parteien zu dokumentieren und zur Verfügung zu stellen. Bestandteil dieser Dokumentation muss eine generelle Strukturbeschreibung des Moduls sein, wohingegen eine umfassende Kenntnis über die detaillierte innere Struktur nicht notwendig ist.

\subsection{Installation, Tests und Inbetriebnahme}

Im Rahmen der Inbetriebnahme eines Moduls gilt es, das vorher gewählte Modul in die bestehende Infrastruktur eines Betriebsstandorts zu integrieren. Hierfür muss es am vorgesehenen Standort aufgestellt und angeschlossen werden. Dies bedarf einer vorherigen Abstimmung über die Integrationstiefe des jeweiligen Moduls in die Anlage. Die rückwirkungsfreie Einbindung in das gesamte System ist dabei sicherzustellen. Die Rückwirkungsfreiheit muss sowohl hinsichtlich des AT-Systems als auch der Versorgung gewährleistet sein.

Der Anschluss an die übergeordnete Infrastruktur erfolgt durch das Verbinden der festgelegten Schnittstellen. Anschließend muss ein Funktionstest des Moduls durchgeführt werden, bevor der Übergang in die Betriebsphase erfolgt. Beim Funktionstest muss auf die Beeinflussung weiterer Module und auf mögliche Rückwirkungen geachtet werden. 
Die für Plug and Produce benötigten Informationen im übergeordneten AS müssen spätestens bei der Inbetriebnahme übertragen werden (in der Regel bereits in der Testphase erforderlich).

Eine spätere Wartung der Module durch den Betreiber ist - je nach ausgehandeltem Wartungsvertrag nur sehr eingeschränkt möglich. Der innere Aufbau eines Moduls ist dem Anwender möglicherweise nicht bekannt oder es ist ihm vertraglich verboten, selbst Wartungsarbeiten durchzuführen. Das bedeutet, dass der Anwender im Falle eines Fehlers auf eine schnelle Reaktion des Modulherstellers angewiesen ist. Andererseits kann bei einem Defekt die Produktion schnell wieder aufgenommen werden, wenn das ausgefallene Modul durch ein baugleiches Modul ersetzt wird.

Bei der Auswahl eines bestimmten Moduls ist der Anwender auf das Know-how des Modulherstellers angewiesen. Dies bedeutet zwar, dass - aus Sicht des Planers - aus der Menge der Hersteller der Beste auf jedem Gebiet ausgewählt werden kann, faktisch wird der Planer jedoch bei der Auswahl der Hersteller aus den oben genannten Gründen einen Kompromiss eingehen.

\subsection{Optimierung}

Der innere Aufbau eines Moduls ist dem Betreiber nicht zwingend bekannt. Außerdem ist es ihm unter Umständen nicht erlaubt, technische Änderungen am Modul vorzunehmen. Dies erschwert die Optimierung auf prozesstechnischer Seite oder macht sie gänzlich unmöglich. Die in einem Modul implementierten Fahrweisen sind ebenfalls nicht zwingend zugänglich. Daher ist es dem Betreiber meist nur möglich, eine Anlage durch die entsprechende Verknüpfung der Module und deren Parametrierung zu optimieren.

Die Effizienz einer World-Scale-Kontianlage wird nicht zu erreichen sein. Verglichen mit einer Mehrzweckanlage lässt sich jedoch die Effizienz steigern, wobei die Flexibilität erhalten bleibt.

\section{HINDERNISSE UND ANFORDERUNGEN}

Während die Schnittstellen zwischen Modulen und übergeordnetem AS im Prinzip bereits vorhanden sind und aktiv in der Industrie genutzt werden (Feldbus, OPC), fehlen im Bereich Software-Engineering und PLT standardisierte Informationsmodelle, um die Integration modularer Automatisierungssysteme auf einfachem Wege durchführen zu können. Aktuelle Entwicklungen bei der Definition von Austauschformaten zwischen Verfahrenstechnik und Automatisierungstechnik zeigen auf, dass die mathematisch-algorithmischen Methoden für ein modulares Automatisierungsengineering zur Verfügung stehen. Beispiele sind die stärkere Formalisierung von Planungsdaten durch die EN 62424 [9], PandIX [10], die Berücksichtigung von Verlässlichkeitsanforderungen in der Prozess- und Ressourcenbeschreibung [11], die Plug-and-Play-Visualisierung für die flexible Automation in der Fertigungs- industrie auf der Basis von EDDL [12] oder die modellgestützte Generierung von Bedienbildern für verschiedene Zielsysteme und Bedienphilosophien aus systemunabhängigen Datenformaten [13].

Das automatisierte Engineering bei Änderungen der Module oder bei der Integration neuer Module in ein übergeordnetes AS ist derzeit jedoch nicht in Sicht. Hier stehen die Systemhersteller vor der Herausforderung, offene Konfigurationsdatenbanken und (Engineering-) Schnittstellen bereitzustellen, um flexibel auf dynamisch veränderte Automatisierungsumgebungen reagieren zu können. In einer Übergangszeit ist der Import von Engineeringdaten der Modullieferanten mit manueller Nachkonfiguration des übergeordneten AS denkbar. Langfristig muss die Zielrichtung zu einer automatisierten Integration mit Plug and Control und Autokonfiguration führen.

Eine weitere Hürde stellt die Zunahme der Schnittstellen dar. Verlangt ein Planer die Möglichkeit, innerhalb eines Moduls in Echtzeit auf Sensordaten Zugriff zu bekommen oder anderweitig ins dortige Leitsystem einzugreifen, muss der Lieferant hierfür eine Schnittstelle anbieten. Im Zuge der Modularisierung wird ein heterogenes Angebot der Module am Markt angestrebt. Dadurch kann sich ungünstigerweise eine große Vielfalt an Kommunikationsschnittstellen zwischen Modulen und übergeordneten Leitsystemen entwickeln. Einen Wald verschiedener Schnittstellen wird ein Anlagenplaner nicht akzeptieren, da die Integration der Module in das gesamte System dadurch sehr aufwändig wird. Aus diesem Grund muss die Schnittstelle zwischen Modul und übergeordneten Systemen formal wie auch technisch standardisiert sein. Denkbar ist beispielsweise die Übertragung eines formalen Anlagenmodells mit Sensorwerten und sonstigen Daten über OPC UA [14]. Eine wesentliche Voraussetzung hierfür wäre es, ein standardisiertes Informationsmodell [15] zu entwickeln, das Planungsdaten, beispielsweise auf Basis von PandIX [10], und Steuerungsdaten, zum Beispiel auf Basis des OPC UA Modells für IEC 61131 [16], integriert darstellt.

Neben der technischen Lösung werden sich darüber hinaus die Beziehungen zwischen Betreibern und Modulherstellern verändern müssen. Die Planer neuer Anlagen werden einen Teil ihrer Aufgaben an die Lieferanten abgeben. Die Planungsaufgabe wird vor allem darin bestehen, die Anforderungen zusammenzufassen und die Module zu einem Gesamtsystem zu integrieren. Dabei wird die Auswahl geeigneter Hersteller und die Aushandlung von Wartungsverträgen eine wichtige Rolle spielen. Die Planer müssen die Hersteller, soweit es der Schutz der eigenen Intellectual Property erlaubt, in die Planung einer Anlage mit einbeziehen, um die bestmögliche Modulauswahl zu treffen beziehungsweise nicht standardisierte Module zu entwickeln. Für die Hersteller bedeutet dies ein höheres Maß an Verantwortung, gibt ihnen aber auch mehr Möglichkeiten, ihre Produkte weiter zu entwickeln und sich im Markt abzuheben, da sie nicht mehr „nur“ Aktoren und Sensoren liefern, sondern ein integriertes Produkt. Auch im Bereich der Anlagenwartung erhalten die Hersteller neue Aufgaben. Die Wartungsarbeiten werden verstärkt von 
Modulherstellern oder deren Vertragspartnern durchgeführt werden. Tritt beim Betrieb einer Anlage ein Problem auf, so wird der Hersteller zumindest an der Lösung beteiligt sein (wenn es sich um ein Problem im Verfahren handelt) oder die Lösung selbst entwickeln müssen (wenn das Problem innerhalb seines Moduls liegt und technischer Natur ist).

Weitere Herausforderungen sind die Themen Knowhow-Schutz von Anwendern und Herstellern sowie ITSicherheitsanforderungen an die Automatisierungstechnik bei den verschiedenen Personengruppen. welche in einer effizient modularisierten Anlage konfigurieren, Wartung durchführen und auf Daten zugreifen.

\section{FAZIT}

Viele technische Anforderungen aus der von der Verfahrenstechnik vorangetriebenen Modularisierung kann die Automatisierungstechnik mit aktuellen Mitteln bereits erfüllen. Für die nahtlose Integration, die Automatisierung der Automatisierung sowie die Erfüllung der aus der Modularisierung resultierenden Schutzziele für IP und IT muss die Automatisierungstechnik Architekturen, Standards und Werkzeuge weiter entwickeln.

\section{AUTOREN}

Prof. Dr.-Ing. LEON URBAS (geb. 1965) ist Inhaber der Professur für Prozessleittechnik an der Technischen Universität Dresden. Seine Hauptarbeitsgebiete beim Engineering verteilter sicherheitskritischer Systeme sind Funktionsintegration, modellgetriebenes Engineering, Modularisierung, Informationsmodelle der Prozessindustrie und Middleware in der Automatisierungstechnik. Einen weiteren Schwerpunkt bildet die Gebrauchstauglichkeit von mobilen Informationssystemen für die Prozessindustrie, Analyse, Gestaltung und Bewertung von Alarmierungs- und Unterstützungssysteme sowie Methoden der Benutzermodellierung zur prospektiven Gestaltung von Mensch-Technik-Interaktion.

TU Dresden,

Institut für Automatisierungstechnik,

01062 Dresden, Tel. +49 (0) 35146339614 ,

E-Mail: leon.urbasatu-dresden.de

Dipl.-Ing. STEPHAN BLEUEL, (geb. 1965) ist Betriebstechnikleiter in einer Produktionsanlage von Sanofi-Aventis. In der IGR (Interessengemeinschaft Regelwerksverfolgung) koordiniert er das Arbeitsfeld Leittechnik. Im Rahmen des Namur-AK 1.12 bearbeitet er die Automatisierung modularer Anlagen und deren zukünftige Entwicklung.

Sanofi-Aventis Deutschland GmbH, Industriepark Hoechst BIdg. G680,

D-65926 Frankfurt am Main, Tel. +49 (0) 6930583096 ,

E-Mail: stephan.bleuelasanofi.com

Dipl.-Wirt.-Ing. TOBIAS JÄGER, (geb. 1984) ist wissenschaftlicher Mitarbeiter am Institut für Automatisierungstechnik der HelmutSchmidt-Universität/Universität der Bundeswehr Hamburg. Seine Arbeitsschwerpunkte sind Modellassoziationen und Abhängigkeitsmanagement im industriellen Lösungsgeschäft für eine effiziente Gestaltung des Engineerings.

Helmut-Schmidt-Universität,

Holstenhofweg 85, D-22043 Hamburg,

Tel. +49 (0) 4065413663 ,

E-Mail: tobias.jaegerRahsu-hh.de
Dipl.-Ing. STEFAN SCHMITZ (geb. 1979) ist PLT-Projektmanager bei der Bayer Technology Services GmbH in Leverkusen. Neben seiner Tätigkeit als PLT-Projektleiter in der Anlagenplanung ist er im $\mathrm{F}^{3}$ Factory Projekt für das Automatisierungskonzept der modularen Demonstrationsanlagen von BTS verantwortlich.

Bayer Technology Services,

Kaiser-Wilhelm-Allee,

D-51368 Leverkusen,

Tel. +49 (0) 2143043275 ,

E-Mail: stefan.schmitz2@abayer.com

Dipl.-Ing. LARS EVERTZ, (geb. 1987) ist wissenschaftlicher Mitarbeiter am Lehrstuhl für Prozessleittechnik der RWTH Aachen University. Seine Arbeitsschwerpunkte sind Automatisierungskonzepte für modulare Anlagen, Apps für die Leittechnik sowie Assistenzsysteme im operativen Anlagenbetrieb.

RWTH Aachen University,

Lehrstuhl für Prozessleittechnik,

Turmstrasse 46, D-52064 Aachen,

Tel. +49 (0) 2418095160 ,

E-Mail: l.evertz@plt.rwth-aachen.de

Dipl.-Ing. TOBIAS NEKOLLA, (geb. 1961) ist PLT-Projektmanager für die internationale Anlagenplanung beim Servicebereich Process Technology \& Engineering der Evonik Industries AG. Zusätzlich hat er leitende Funktionen im PLS-Fachreferat des Servicebereichs.

Evonik Industries,

TE-EN-E-A2, Rodenbacher Chaussee 4,

D-63457 Hanau-Wolfgang, Tel. +49 (0) 6181594043 ,

E-Mail: tobias.nekollađevonik.com 
DANKSAGUNG

Bei der Erstellung der Vorträge des AK 1.12 auf der Namur-Hauptsitzung 2011, die dieser Artikel zusammenfasst, haben die Mitglieder des Namur-AK 1.12 Stephan Bleuel (Sanofi-Aventis), Andreas Bamberg (Merck), Tobias Nekolla (Evonik Industries) Tobias Jäger (HSU-HH), Hisham Mubarak (BASF), Lars Evertz (RWTH-Aachen), Leon Urbas (Technische Universität Dresden); Markus Vogel (Celanese), Stefan Schmitz (Bayer Technology Services) und Ulf Clausnitzer (Merck) mitgewirkt.

\section{REFERENZEN}

[1] Bott, T., Schembecker, G.: Die 50 \%-Idee - Vom Produkt zur Produktions $\neg$ anlage in der halben Zeit. Tandemvortrag ProcessNet Jahrestreffen 8. - 10.9.2009, Mannheim

[2] Hady, Ł.; Lüneburg, W.; DylĐg, M.; Wozny, G. „Modular investment cost estimate of multipurpose chemical plant", Chemical and Process Engineering 28, 1, S. 17-31. 2007

[3] F3-Projekt. www.f3factory.com

[4] IEC 61512-1: Chargenorientierte Fahrweise Teil 1: Modelle und Terminologie. 1996

[5] Obst, M., Urbas, L.: Integriertes modulares Engineering. PAT Jahrestagung, Fulda

[6] ATEX-Produktrichtlinie 94/9/EG

[7] DIN EN 61346 Strukturierungsprinzipien und Referenzkennzeichnung von industriellen Systemen, Anlagen, Ausrüstungen und Industrieprodukten.

[8] IEC 62443: Industrial communication networks - Network and system security. 2010

[9] IEC 62424: Specification for Representation of process control engineering requests in $P \& I$ Diagrams and for data exchange between P\&ID tools and PCE-CAE. 2007

[10] Epple, U., Remmel, M., Drumm, O.: Modellbasiertes Format für RI-Informationen. atp - Automatisierungstechnische Praxis 53, 1-2, S. 14-26

[11] Opgenoorth, B., Richter, J.H., Grosch, T., Wolff, D., Fay, A. Verlässlichkeitsanforderungen in der Prozess- und Ressourcenbeschreibung. atp - Automatisierungstechnische Praxis 53, 3, S. 44-53

[12] Brecher, C., Kolster, D., Herfs, W., Jensen, S., Pleßow, M.: Plug-and-Play-Visualisierung für flexible Automation. atp - Automatisierungstechnische Praxis 53, 4, S. 42-49

[13] Doherr, F., Drumm, O., Franze, V., Urbas, L.: Bedienbilder auf Knopfdruck. atp - Automatisierungstechnische Praxis $53,1-2$, S. $14-26$

[14] W. Mahnke, S.-H.Leitner, M. Damm: OPC Unified Architecture, Springer, 2009

[15] W. Mahnke, A., Gössling, M. Graube, L. Urbas: Information modeling for middleware in automation. Tagungsband 16 th IEEE Conference on Emerging Technologies \& Factory Automation (ETFA2011). doi:10.1109/ETFA.2011.6059111

[16] PLCOpen: OPC UA Information Model for IEC 61131-3, Release $1.00,2010$

\section{Herausforderung}

Automatisierungstechnik

Mit dem atp-award werden zwei Autoren der atp edition für hervorragende Beiträge ausgezeichnet. Ziel dieser Initiative ist es, Wissenschaftler und Praktiker der Automatisierungstechnik anzuregen, ihre Ergebnisse und Erfahrungen in Veröffentlichungen zu fassen und die Wissenstransparenz in der Automatisierungstechnik zu fördern. Teilnehmen kann jeder Autor der zum Zeitpunkt der Veröffentlichung nicht älter als 35 Jahre ist. Nach Veröffentlichung eines Beitrags ist der Autor, wenn er die Bedingung erfüllt, automatisch im Pool. Die Auswahl des Gewinners übernimmt die atp-Fachredaktion. Derjenige Autor, der im Autorenteam der jüngste ist, erhält stellvertretend für alle Autoren die Auszeichnung. Der Preis wird in zwei Kategorien ausgelobt: Industrie und Hochschule. Die Kategorienermittlung ergibt sich aus der in dem Beitrag angegebenen Adresse des jüngsten Autors.

Veröffentlichungen - Beitrag zum Wissenspool im Fachgebiet Automatisierungstechnik

Die Entwicklung eines Wissensgebietes erfolgt durch einen kooperativen Prozess zwischen wissenschaftlicher Grundlagenforschung, Konzept- und Lösungsentwicklung und Anwendung in der Praxis. Ein solcher Prozess bedarf einer gemeinsamen Informationsplattform. Veröffentlichungen sind die essentielle Basis eines solchen Informationspools. Der atp-award fördert den wissenschaftlichen Austausch im dynamischen Feld der Automationstechnik. Nachwuchsingenieure sollen gezielt ihre Forschungen präsentieren können und so leichter den Zugang zur Community erhalten. Der Preis ist mit einer Prämie von jeweils 2000€ dotiert.

\section{Die Auswahl erfolgt in zwei Stufen:}

Voraussetzung für die Teilnahme ist die Veröffentlichung des Beitrags in der atp edition. Jeder Aufsatz, der als Hauptbeitrag für die atp edition eingereicht wird, durchläuft das Peer-Review-Verfahren. Die letzte Entscheidung zur Veröffentlichung liegt beim Chefredakteur. Wird ein Beitrag veröffentlicht, kommt er automatisch in den Pool der atp-awardBewerber, vorausgesetzt einer der Autoren ist zum Zeitpunkt der Veröffentlichung nicht älter als 35 Jahre. Ausgezeichnet wird der jüngste Autor stellvertretend für alle Autoren der Gruppe. Eine Jury aus Vertretern der atp-Fachredaktion und des -Beirats ermittelt schließlich den Gewinner in den jeweiligen Kategorien Hochschule und Industrie. Der Rechtsweg ist ausgeschlossen.

\section{Beiträge richten Sie bitte an:}

Oldenbourg Industrieverlag $\mathrm{GmbH}$ Herrn Prof. Leon Urbas

Chefredakteur atp edition/automatisieren! by atp Rosenheimer Straße 145 • 81761 München Tel. +49 (0) 8945051418 • E-Mail: urbasविoiv.de

Beachten Sie die Autorenhinweise der atp edition für Hauptbeiträge unter folgendem Link: http://www.atp-online.de 